\title{
Clinical Practice Guidelines and Experts' Consensuses for Treatment of Coronavirus Disease 2019 (COVID-19) Patients with Chinese Herbal Medicine: A Systematic Review*
}

\author{
LI Yu-xi ${ }^{1}$, LI Juan ${ }^{2}$, ZHANG Yue ${ }^{2}$, TIAN Yan-ping², ZHANG Yong-gang ${ }^{3}$, \\ JIN Rong-jiang ${ }^{2}$, GUO Yan ${ }^{4}$, and Mike Clarke ${ }^{5}$
}

\begin{abstract}
Objective: To systematically review the clinical practice guidelines for the treatment of coronavirus disease 2019 (COVID-19) patients with Chinese herbal medicine (CHM), summarize their recommendations and evaluate their qualities. Methods: Electronic databases, websites of governments, academic associations or organizations for eligible guidelines were searched up to April 2020. Information were extracted to summarize recommendations for the use of Chinese herbal formulae and Chinese patent medicine (CPM) for COVID-19 patients. The methodological quality and reporting quality of the included guidelines were independently evaluated, using the Appraisal of Guidelines for REsearch and Evaluation (AGREE) II tool and Reporting Items for Practice Guidelines in healthcare (RIGHT) statement, respectively. Results: Totally 45 guidelines were identified: 42 (93\%) suggested specific CM formulae for treating COVID-19 patients; and 35 (78\%) assigned patients diagnosed with COVID-19 to 4 stages (early, development, critical and recovery). The recommended formulae and CPMs for each stage with diverse syndrome types have been summarized. Most of the included guidelines were of low methodological quality, with only one achieving moderate quality of AGREE II. The frequency with which the 35 items in the Reporting Items for Practice Guidelines in Healthcare (RIGHT) checklist were reported averaged 36.5\% across the 45 guidelines. Conclusions: This systematic review has summarized current recommendations for treating COVID-19 patients with $\mathrm{CHM}$ and shows that the general quality of present guidelines is quite low. The development of future guidelines should include increased awareness of the features of good quality guidelines.
\end{abstract}

KEYWORDS coronavirus disease 2019, COVID-19, Chinese medicine, herbal medicine, clinical practice guideline, systematic review

Coronavirus disease 2019 (COVID-19) has rapidly spread throughout the world since December 2019, and it was declared as a pandemic by the World Health Organization (WHO) on 11 March, 2020. ${ }^{(1,2)}$ Infections have been found in more than 200 countries, areas or territories and more than 6 million people were confirmed with COVID-19, up to June 2020. ${ }^{(3)}$ COVID-19 is caused by severe acute respiratory syndrome coronavirus 2 (SARS-CoV-2), which induces inflammation and infiltration in lungs and can lead to systemic inflammatory cytokine storms. ${ }^{(4,5)}$ As of June 2020, no vaccine or specific effective drugs have been identified for COVID-19, and supportive treatments are mainly used to manage its symptoms.

Through its thousands of years' history of development, Chinese medicine (CM) has acquired abundant clinical experiences and efficacy in the prevention and treatment of epidemics and endemic diseases. In CM, COVID-19 pertains to a type of "plague" which is caused by evil qi. The characteristics of the evil qi include cold, dampness, and toxin. According to $\mathrm{CM}$ theory, Wuhan as center of the epidemic is a city with many lakes and rivers that has high humidity. The climate of Wuhan suggests a relationship with dampness, and patients diagnosed with COVID-19 often have gastrointestinal symptoms such as nausea, vomiting, abdominal distension, and

(C)The Chinese Journal of Integrated Traditional and Western Medicine Press and Springer-Verlag GmbH Germany, part of Springer Nature 2020

*Supported by Education Fund of Chengdu University of Traditional Chinese Medicine

1. School of Acupuncture-Moxibustion and Tuina, Chengdu University of Traditional Chinese Medicine, Chengdu (610032), China; 2. School of Health Preservation and Rehabilitation, Chengdu University of Traditional Chinese Medicine, Chengdu (610032), China; 3. West China Hospital, Sichuan University, Chengdu (610032), China; 4. Xiyuan Hospital, China Academy of Chinese Medicine Sciences, Beijing (100091), China; 5. Northern Ireland Clinical Trials Unit and Methodology Hub, Centre for Public Health, Queen's University Belfast, Belfast, UK Correspondence to: Prof. GUO Yan, E-mail: guoyan0314@126. com; Prof. JIN Rong-jiang, E-mail: cdzyydxjrj@126.com; Prof. Mike Clarke, E-mail:m.clarke@qub.ac.uk

DOI: https://doi.org/10.1007/s11655-020-3431-x 
diarrhea, which also relates to dampness. Therefore, in $\mathrm{CM}$, the combination of the outbreak location and clinical manifestations of COVID-19 patients, shows that the onset of COVID-19 is mostly caused by pathogen of dampness. ${ }^{(6)}$ This leads to the suggestion that herbs with functions of promoting Fei (Lung) and expelling dampness can be applied, and the use of $\mathrm{CM}$ has been associated with benefits against COVID-19. ${ }^{(7,8)}$ According to the State Council Information Office of China, more than 70,000 COVID-19 patients (91.5\% of total confirmed COVID-19 cases in China) were treated with Chinese herbal medicine (CHM) with promising effects. ${ }^{(9)}$ The National Health Commission of China released the Diagnosis and Treatment Guideline of Novel Coronavirus Pneumonia (Edition 7) in March 2020, which contains recommendations for using CM for COVID-19 patients, based on different stages and syndrome types of the disease. ${ }^{(10)}$ Furthermore, provinces, cities, and autonomous regions of China also issued clinical practice guidelines recommending $\mathrm{CHM}$.

This study provides a summary and appraisal of these guidelines, using systematic review methods. We systematically retrieved clinical practice guidelines for the treatment of COVID-19 patients with CHM, summarized the recommended CHMs for COVID-19 with stages and syndrome types, and evaluated the methodological quality and reporting quality of the included guidelines.

\section{METHODS}

\section{Study Registration}

This systematic review is reported in accordance with the Preferred Reporting Items for Systematic Reviews and Meta-Analysis (PRISMA) guidance. ${ }^{(11)}$ The study protocol was registered in the International Prospective Register of Systematic Reviews (No. CRD42020179205) and has been published. ${ }^{(12)}$ The full PRISMA checklist and flow diagram are in Appendix 1.

\section{Databases and Search Strategy}

The following databases were searched, including PubMed, Embase, Chinese Biomedical Literature Database (CBM), China National Knowledge Infrastructure (CNKI), Chinese Science and Technology Periodical Database (VIP), and Wanfang database from inception to April 2020. We also supplemented these searches by searching sources of guidelines including the National Guideline Clearinghouse (NGC), Guidelines International Network (GIN), National Institute for Health and Clinical Excellence (NICE), Scottish Intercollegiate Guidelines Network (SIGN) and WHO. The websites of governments, academic associations or organizations for grey literature were also searched. Considering that consensus is mostly published by academic associations, the search of websites of academic associations was added on the basis of the original protocol. The searches used a combination of guideline, coronavirus and $\mathrm{CM}$ terms. The detailed search strategy of PubMed is described in Appendix 2, and suitably modified search strategies were applied to the other sources.

\section{Eligibility Criteria}

Clinical practice guidelines, expert consensus statements and guidance documents which provided information on using CHM for COVID-19 patients were included. To be eligible, the guidelines should be published after December 2019 by any advising body or healthcare organization. Older versions of included guidelines or consensus statements were excluded.

\section{Study Selection and Data Extraction}

Two reviewers ( $L Y X$ and $Z D L$ ) independently identified potentially eligible guidelines according to predetermined criteria. Disagreements were discussed and arbitrated by a third reviewer (ZYG). In order to achieve consistency in extracted items, the data extraction process was piloted using a sample of eligible guidelines and the Kappa coefficient was evaluated. A kappa value of $<0.2$ indicates poor, $0.21-0.40$ means fair, $0.41-0.60$ means moderate, $0.61-0.80$ means good and 0.81-1.00 means very good agreement. ${ }^{(13)}$ Two reviewers (LJ and ZY) independently extracted the following data: (1) characteristics of guidelines: name, type, source and date; (2) stage of disease; (3) syndrome type; (4) treatment principle; (5) recommended formula; and (6) recommended Chinese patent medicine (CPM), etc. Disagreements were resolved by discussion among the review team.

\section{Quality Assessment}

The methodological quality and reporting quality of the included guidelines were evaluated using the Appraisal of Guidelines for REsearch and Evaluation (AGREE) II tool ${ }^{(14)}$ and Reporting Items for Practice Guidelines in Healthcare (RIGHT) statement, ${ }^{(15)}$ respectively (see below). To ensure at least $80 \%$ consistency in quality assessments, we pre-assessed a sample of eligible guidelines and discussed the results among reviewers. The kappa coefficient was also evaluated. Subsequently, 2 reviewers ( $L Y X$ and $L J$ ) independently appraised the quality of each included guideline. Discrepancies were discussed and resolved among the reviewers. 
The AGREE II instrument is a tool that can be used to assess the methodological rigour and transparency in the development of a guideline. ${ }^{(13,16)}$ It consists of 23 items in 6 domains: (1) scope and purpose (items 1-3), (2) stakeholder involvement (items 4-6), (3) rigour of development (items 7-14), (4) clarity of presentation (items 15-17), (5) applicability (items 18-21) and (6) editorial independence (items 22-23). Each item is rated from 1 (did not meet criteria) to 7 (met criteria). The RIGHT statement focuses on the essential items for reporting guidelines, which can assist developers in reporting their guideline and helps readers to get a better understanding of the guideline. It contains 22 items in 7 domains: (1) basic information (items 1-4), (2) background (items 5-9), (3) evidence (items 10-12), (4) recommendations (items 13-15), (5) review and quality assurance (items 16-17), (6) funding, declaration and management of interest (items 18-19), and (7) other information (items 20-22).

\section{Data Analysis}

Microsoft Excel 2019 was used to summarize and analyze the recommendations for treating COVID-19 patients with CM formulae and CPM, as well as the results of quality assessment.

\section{RESULTS}

A total of 275 records in electronic databases and 26 records on websites of governments and organizations were identified. After careful assessment, 45 of these met the inclusion criteria and were included for analysis (Appendix 2). ${ }^{(10,17-60)}$

\section{Characteristics of Guidelines}

The characteristics of the 45 included guidelines are presented in Appendix 3. Two guidelines focused on recommendations for children and 2 focused on people over 60 years of age. Of the 45 guidelines, 42 (93\%) contained a treatment plan for COVID-19 patients and $3(7 \%)$ only covered prevention protocols for COVID-19. Thirty-five (78\%) guidelines staged the disease, $39(87 \%)$ classified syndromes based on different clinical manifestations of patients, 43 (96\%) detailed the formula of herbs, 17 (38\%) offered adjustments of formula based on different symptoms, 37 (82\%) suggested dosage of each herb, 25 (56\%) mentioned decocting methods of herbs, $25(56 \%)$ recommended administration and 32 (71\%) suggested CPM. Twenty-seven (60\%) guidelines were issued by a national or provincial health commissions or administrations of CM of China, 13 (29\%) were published by expert groups and $5(11 \%)$ were suggested by academic associations or centers of CM. Some guidelines suggested detailed formulae without prescription names, which are not conducive for summary and promotion. The principles, names of formulae and CPMs for patients with different syndromes were summarized.

\section{Prevention Plan}

Twenty-one guidelines offered prevention plans for COVID-19, of which 13 directly listed the prevention formulae or CPMs. The remainder presented suggestions for different groups of people.

For people with yang deficiency constitution, the principle of prevention is supplementing qi and strengthening the surface, dispelling cold and removing dampness, and the recommended CPM is Yupingfeng Powder (玉屏风散). For people with yin deficiency constitution, supplementing qi and promoting the production of body fluid, clearing fire and relieving pharynx is the principle of prevention, and the recommended CPMs are Shenmai Oral Liquid (参麦口服 液), Yangshen Chongcao Capsule (洋参虫草胶囊). People with weak constitution and old people are recommended with Guizhi Decoction (桂枝汤), Yupingfeng Powder and Shenzhu Powder (神术散) as formulae of herbs and Huoxiang Zhengqi Pill/Oral Liquid (雚香正气丸/口服液), Yinhuang Qingfei Capsule (银黄清肺胶囊), Qingre Jiedu Granules (清热解毒颗 粒) as CPMs to harmonize Ying (nutrient) and Wei (defense), replenish qi and solidify exterior.

Prevention Program for Pneumonia of Influenza and COVID-19 in Shandong Province also suggested prevention principles for different population groups: reinforcing qi and nourishing yin, strengthening body and consolidating exterior for general population; nourishing yin and clearing heat, regulating qi and strengthening vital energy for pregnant women; clearing heat and detoxicating, supplementing qi and consolidating exterior for children; invigorating qi and $\mathrm{Pi}$ (Spleen), nourishing yin and moistening lung for elderly; reinforcing qi and nourishing yin, strengthening body and consolidating exterior for people in close contact with COVID-19 patients and healthcare workers. For patients with comorbid chronic diseases, prevention principles are as follows: clearing heat and generating fluid, nourishing yin and consolidating exterior for diabetic patients; clearing heat and nourishing yin, calming Gan (Liver) and consolidating exterior for hypertensive patients; reinforcing qi and nourishing yin, clearing heat and consolidating exterior for patients with coronary heart diseases; reinforcing qi to invigorate $\mathrm{Pi}$, clearing heat and consolidating exterior for patients with chronic respiratory diseases. 


\section{Medical Observation Period for People without} Confirmed Diagnosis of COVID-19

Some patients in the medical observation period may have some flu-like symptoms without a confirmed diagnosis of COVID-19, and CPMs are mostly recommended for patients in this period. Huoxiang Zhengqi Capsule (Pill, Liquid, Oral liquid) is recommended for adults and children with fatigue and gastrointestinal discomfort to dispel dampness in exterior and regulate qi and Wei (Stomach). For adults with fatigue and fever, Jinhua Qinggan Granule (金花清感颗粒), Lianhua Qingwen Capsule/Granule (连 花清瘟胶囊/颗粒), Shufeng Jiedu Capsule/Granule (疏风 解毒胶囊/颗粒), Fangfeng Tongsheng Pills/Granules (防 风通圣丸/颗粒), Antiviral Oral Liquid (抗病毒口服液) are suggested to expel wind and dampness, clear away heat and detoxify. Chaishi Tuire Granules (柴石退热颗粒), Kegan Liyan Oral Liquid (克感利咽口服液), Lanqin Oral Liquid (蓝芩口服液), Xiaochaihu Granules (小柴胡颗粒), Vitamnin C Yinqiao Tablet (维C银趐片) and Qingkailing Capsule (清开灵胶震) are also advised to adults in Guangdong Province. Children with same symptoms can be treated with Lianhua Qingwen Capsules/ Granules, Xiaoer Qingqiao Granules (小儿青趐颗粒) and Xiaoer Jiegan Granules (小儿解感颗粒). Jinlian Qingre Effervescent Tablets (金莲清热泡腾片), Xiaoer Chiqiao Qingre Granules (小儿豉翅清热颗粒) and Jinye Baidu Granules (金叶败毒颗粒) are advised when constipation is accompanied by fatigue and fever in children.

\section{Clinical Treatment Period for Confirmed COVID-19 Cases}

As noted above, in CM theory, the main pathogens of COVID-19 are dampness and toxin. However, as COVID-19 disease develops in a patient, different syndrome types may evolve. For this review, the syndrome types that are mentioned at least twice in guidelines were summarized (Appendix 4).

In accordance with the nature of COVID-19, Qingfei Paidu Decoction (清肺排毒汤) is recommended in most of the included guidelines for mild, moderate and severe cases, and can be used reasonably with the consideration of the actual conditions of critically sick patients. Qingfei Paidu Decoction is composed of several classic prescriptions in Shang Han Za Bing Lun (Treatise on Cold Pathogenic and Miscellaneous Diseases) written by ZHANG Zhong-jing in the Han Dynasty. According to Prof. XUE Bo-shou, a CM Master, this prescription is an innovative combination that has effects of dispelling colddampness and regulating organs. ${ }^{(61)}$

\section{Early Stage}

Based on CM theory, in the early stage of COVID-19, the exterior and Fei of the patient is attacked by evil qi, which includes cold-dampness, dampness-heat, heat-toxin or warm pathogen. The treatment principle during this stage is clearing and removing the pathogen, while promoting Fei at the same time. For dispersing cold-dampness, Huoxiang Zhengqi Powder and Lianhua Qingwen Capsule are the most recommended formulae and CPM.

\section{Development Stage}

In the development stage of COVID-19, the evil qi gradually invades and attacks the lung, which leaves the obstruction of toxin, dampness-toxin or dampnessheat in the lung. With the treatment principle of clearing evil qi, Xiyanping Injection (喜炎平注射液), Xuebijing Injection (血必净注射液), Reduning Injection (热毒宁注射 液) and Tanreqing Injection (痰热清注射液) are the most recommended CPMs.

\section{Critical Stage}

In CM theory, after the evil qi cracked the system of Wei, it constantly attacks Ying, which stores the nutrient substances and mind of the body. This means that the disease has entered a critical stage of fighting between healthy qi and evil qi. The treatment principle of this stage is mainly clearing the evil qi and supplementing healthy qi. The recommended formulae are diverse, based on different syndrome types, and Xiyanping Injection, Xuebijing Injection, Reduning Injection, Tanreqing Injection and Xingnaojing Injection (醒脑净注射液) are recommended.

\section{Recovery Stage}

In CM theory, when the healthy qi of the human body defeats the evil qi, the process of disease finally moves to the recovery stage. In this stage, a lot of blood and qi is consumed, and one of the syndrome types is deficiency of both qi and yin pattern. Shengmai Decoction/Granule/Oral Liquid/Injection（生脉汤/口服液 (注射液) is the most recommended CM for this pattern. When patients manifest mostly symptoms related to Fei and $\mathrm{Pi}$ (e.g. shortness of breath, cough, low food intake and abdominal distention), the syndrome type is Fei-Pi qi deficiency pattern, and Liujunzi Decoction (六君子汤), Shengling Baizhu Powder (参苓白术散) are suggested.

\section{Quality Assessment of Included Guidelines}

The kappa values for our independent assessments of the included guidelines using the AGREE II checklist and RIGHT checklist ranged from 0.73 to 0.82 , indicating 
good consistency for quality assessments.

\section{Methodological Quality}

Using AGREE II, we rated only one of the 45 included guidelines as moderate quality (achieving 63\%). The others were all of low quality, with scores from $18 \%$ to $44 \%$. The score of each domain of AGREE II and the overall quality of each included guideline are summarized in Appendix 5. Domain 4 (Clarity of presentation) achieved the highest mean score at $72 \%$ (range $28 \%$ to $83 \%$ ), followed by domain 1 (Scope and purpose) with 69\% score (44\% to $83 \%$ ). Domains 3 (Rigour of development) and 6 (Editorial independence) obtained the lowest scores of $5 \%$ and $4 \%$, respectively.

\section{Reporting Quality}

Overall, the average report rate of the 35 items in the RIGHT checklist in the included guidelines was $36.5 \%$. For the 35 items in RIGHT checklist, the number of guidelines that reported the item ranged from $0(0 \%)$ for 7 items to all 45 (100\%) for 2 items (Appendix 6). The most adequately reported items in the RIGHT statement were items 1a [Identify the report as a guideline, that is, with "guideline(s)" or "recommendation(s)" in the title] and 1c (Describe the focus of the guideline, such as screening, diagnosis, treatment, management, prevention or others), which were reported in all included guidelines. It was followed by item 7a [Describe the primary population(s) that is addressed by the recommendation(s) in the guideline] and 13a (Provide clear, precise, and actionable recommendations), which were reported in 44 (98\%) and $43(96 \%)$ guidelines, respectively. The items that were not reported in any guideline were $11 \mathrm{~b}$ (If the guideline developers used existing systematic reviews, reference these and describe how those reviews were identified and assessed (provide the search strategies and the selection criteria, and describe how the risk of bias was evaluated) and whether they were updated), 13c (Indicate the strength of recommendations and the certainty of the supporting evidence), 14a [Describe whether values and preferences of the target population(s) were considered in the formulation of each recommendation], 14b (Describe whether cost and resource implications were considered in the formulation of recommendations), 16 (Indicate whether the draft guideline underwent independent review), 17 (Indicate whether the guideline was subjected to a quality assurance process) or 18 a [Describe the role of funder(s) in the different stages of guideline development and in the dissemination and implementation of the recommendations].

\section{DISCUSSION}

This study comprehensively summarizes the current clinical practice recommendations for CHM for COVID-19 patients based on the 45 included guidelines, available up to April 2020. Among these guidelines, 42 (93\%) suggested specific CM formulae for treating COVID-19, and 35 (78\%) divided patients with COVID-19 into 4 stages (early, development, critical and recovery). Most of the included guidelines were of low methodological quality, with only one rated as moderate quality using AGREE II. The frequency with which the 35 items in the RIGHT checklist were reported averaged $36.5 \%$ across the 45 guidelines. The rapid guideline conducted by Jin, et $\mathrm{al}^{(17)}$ reached the highest report rate, with $69 \%$ of the items reported.

At present, emerging evidence suggests promising effects of CHM for COVID-19 patients and this is reflected in the published guidelines. In the early stage of treatment, Huoxiang Zhengqi Powder and Lianhua Qingwen Capsule are most recommended for promoting Fei to expel pathogens. ${ }^{(17,24,34,45-47,57)}$ Several clinical observational studies have demonstrated a significant improvement with Lianhua Qingwen Granules combined with Western medicine in the relief of the symptoms of patients suspected of having COVID-19, such as fever, cough, fatigue and shortness of breath. ${ }^{(62-64)}$ A prospective multicenter openlabel randomized trial with 284 participants which compared conventional treatment alone versus conventional treatment in combination with Lianhua Qingwen Capsules showed that rate of recovery ( $91.5 \%$ vs. $82.4 \%, P=0.022)$, improvement in chest computed tomographic manifestations $(83.8 \%$ vs. $64.1 \%, P<0.001)$ and clinical cure $(78.9 \%$ vs. $66.2 \%$, $P=0.017$ ) were higher in the treatment group, and the median time to symptom recovery was markedly shorter (median: 7 vs. $10 \mathrm{~d}, P<0.001) .{ }^{(5)}$ A systematic review and meta-analysis with 4 randomized trials and 7 controlled clinical trials found Lianhua Qingwen Granules shortened the duration of fever $(P=0.01)$, fatigue $(P=0.02)$ and cough $(P=0.038)$ in COVID-19 patients. ${ }^{(65)}$ Qingfei Paidu Decoction is recommended in Chinese National Guideline of Diagnosis and Treatment Protocol for COVID-19 (Trial Version 7) and other guidelines for mild, moderate and severe COVID-19..$^{(10,20,23,24,32,49,51)}$ In a clinical observational study with 98 patients in Sichuan Province, treatment with Qingfei Paidu Decoction indicated a positive therapeutic effect. After 3 days of treatment, the levels of lymphocyte, AST, ALT and D-dimer were recovered to normal $(P<0.01)$. After 6 days of treatment, C-reactive protein and erythrocyte sedimentation rate recovered to normal $(P<0.01)$. After 
9 days, over $90 \%$ of the patients' laboratory indexes became normal. CT image results showed 79 patients were improved after 6 days of treatment. ${ }^{(66)}$ Furthermore, Luo, et $\mathrm{al}^{(67)}$ found that blood samples of 54 confirmed COVID-19 patients from Hubei hospitals showed an increase of number of leukocytes, neutrophil, lymphocyte and platelet, while the numbers of erythrocytes, hemoglobin concentration and hematocrit decreased after treatment with Qingfei Paidu Decoction. A recent pilot randomized trial assessed outcomes in 42 severe cases of patients with COVID-19 treated with either China guideline-based formulae plus standard care or standard care alone. Results showed the odds of death were non-significantly lower in the combined therapy group than in the standard care group (odds ratio $0.59,95 \%$ confidential interval 0.148 to 2.352, $P=0.454) .{ }^{(68)}$ Although these results are preliminary in the context of the global COVID-19 pandemic and need assessment in a formal systematic review, ${ }^{(69)}$ they provide useful information for the guideline-based $\mathrm{CHM}$ treatment of patients with COVID-19, by suggesting that $\mathrm{CHM}$ may be clinically effective and is worthy of further investigation.

The methodological and reporting quality of most of the included guidelines was low. According to AGREE II, domains 3 (Rigour of development) and 6 (Editorial independence) obtained the lowest scores. Most of the included guidelines neither used a systematic search to collect their evidence, nor describe the process of formulation of recommendations or report the conflict of interests. The results from our use of the RIGHT checklist were consistent with those for AGREE II. The methods used for developing recommendations and quality assessment, and the funding source were reported least. These shortcomings are important because guidelines need to be of high quality if they are to influence clinical practice for the better, and guidelines with poor quality may mislead clinicians. However, there are some reasons that may account for this situation. First, COVID-19 is a very urgent public health issue requiring rapid responses from national authorities at all levels, which means that organizations might omit some of the formal processes of developing recommendations in order to publish the guidelines faster. Second, among the guidelines reviewed, $26(58 \%)$ guidelines were issued by national or provincial health committee or administrations of CM of China, and there may be no formal funding body to declare. However, it is still clear that some guidelines, such as those published by expert groups or associations, need to improve their methodological and reporting quality to allow them to meet the standards of AGREE II and RIGHT.
To achieve a solid evidence, future research should follow the AGREE II and RIGHT checklist during the guideline development. ${ }^{(70)}$ Recently, Ang, et $\mathrm{al}^{(71,72)}$ published two rapid reviews of guidelines of herbal medicine for COVID-19. We have improved on those by systematically searching and retrieving more guidelines relating to the recommendation of CHM for COVID-19 patients based on CM theory. We also conducted this systematic review with prior study design and registration, and independently evaluated each included guideline against recognized standards for practice guidelines: AGREE II for methodological quality and RIGHT for reporting quality. These findings provide useful insights for future guideline developers and people studying guidelines and guideline development.

This study has some limitations: (1) we only searched Chinese and English databases, so our findings may not apply to guidelines in other languages; (2) we summarized formulae and CPMs if they were originated from Chinese classic medical books with prescription names and if they were recommended in more than 2 guidelines, which means that there may be some missing information, such as prescriptions based on local doctors' experiences or recommendations in a single guideline; (3) the methodological and reporting quality of most of included guidelines was low, which may influence the certainty of our results.

In conclusion, this study has summarized current recommendations for the use of CHM to prevent COVID-19 and to treat it during the medical observation period and clinical treatment period; including in the early, development, critical and recovery stages. However, the general quality of present guidelines is low and the development of future guidelines should include increased awareness of the features of good quality guidelines.

\section{Conflict of Interest}

The authors declare that they have no competing interests. The funder has no role in developing this article.

\section{Author Contributions}

Clarke M, Jin RJ and Guo Y designed the study. Li YX, Li $J$ and Zhang $Y G$ drafted the manuscript. Tian $Y P$ and Zhang $Y$ searched the literature. Li YX, Tian YP and Zhang $Y$ analyzed the data. $\mathrm{Li} \mathrm{YX}$, Li J and Zhang $\mathrm{Y}$ contribute equally to this work. All authors approved the manuscript.

Electronic Supplementary Material: Supplementary material (Appendix 1-6) is available in the online version of this article at https://doi.org/10.1007/s11655-020-3431-x. 


\section{REFERENCES}

1. Munster VJ, Koopmans M, van Doremalen N, et al. A Novel Coronavirus Emerging in China-Key Questions for Impact Assessment. N Engl J Med 2020;382:692-694.

2. Zhou T, Liu Q, Yang Z, et al. Preliminary prediction of the basic reproduction number of the Wuhan novel coronavirus 2019-nCoV. J Evid Based Med (Chin) 2020;13:3-7.

3. Wu Z, McGoogan JM. Characteristics of and important lessons from the coronavirus disease 2019 (COVID-19) outbreak in China: summary of a report of 72314 cases from the Chinese Center for Disease Control and Prevention. JAMA 2020;323:1239-1242.

4. Huang $C$, Wang $Y, L i X$, et al. Clinical features of patients infected with 2019 novel coronavirus in Wuhan, China. Lancet 2020;395:497-506.

5. Hu K, Guan WJ, Bi Y, et al. Efficacy and safety of Lianhuaqingwen capsules, a repurposed Chinese herb, in patients with coronavirus disease 2019: a multicenter, prospective, randomized controlled trial. Phytomedicine 2020:153242.

6. Wang SX, Wang Y, Lu YB, et al. Diagnosis and treatment of novel coronavirus pneumonia based on the theory of traditional Chinese medicine. J Integr Med 2020;18:275-283.

7. Ren JL, Zhang AH, Wang XJ. Traditional Chinese medicine for COVID-19 treatment. Pharmacol Res 2020;155:104743.

8. Luo $\mathrm{E}$, Zhang $\mathrm{D}$, Luo $\mathrm{H}$, et al. Treatment efficacy analysis of traditional Chinese medicine for novel coronavirus pneumonia (COVID-19): an empirical study from Wuhan, Hubei Province, China. Chin Med 2020;15:34.

9. Novel Coronavirus Pneumonia Emergency Response Epidemiology Team. The epidemiological characteristics of an outbreak of 2019 novel coronavirus diseases (COVID-19) in China. Chin J Epidemiol (Chin) 2020;41:145-151.

10. National Administration of Traditional Chinese Medicine. Diagnosis and treatment guideline of novel coronavirus pneumonia (Edition 7). China 2020. Available at: http://ghs.satcm.gov.cn/ gongzuodongtai/2020-03-20/14089.html.

11. Moher D, Liberati A, Tetzlaff $\mathrm{J}$, et al. Preferred reporting items for systematic reviews and meta-analyses: the PRISMA statement. BMJ 2009;339:b2535.

12. Li Y, Li J, Zhong D. et al. Clinical practice guidelines and experts' consensuses of traditional Chinese herbal medicine for novel coronavirus (COVID-19): protocol of a systematic review. Syst Rev 2020;9:170.

13. Altman DG. Practical Statistic for Medical Research. London: Chapman and Hall; 1990.

14. Brouwers MC, Kho ME, Browman GP, et al. AGREE II: advancing guideline development, reporting, and evaluation in health care. Prev Med 2010;51:421-424.

15. Chen $\mathrm{Y}$, Yang $\mathrm{K}$, Marušic $\mathrm{A}$, et al. A reporting tool for practice guidelines in health care: the RIGHT statement. Ann Intern Med 2017;166:128-132.

16. Simancas-Racines D, Montero-Oleas N, Vernooij RWM, et al. Quality of clinical practice guidelines about red blood cell transfusion. J Evid Based Med 2019;12:113-124.

17. Jin $\mathrm{YH}$, Cai L, Cheng ZS, et al. A rapid advice guideline for the diagnosis and treatment of 2019 novel coronavirus (2019-nCoV) infected pneumonia (standard version). Mil Med Res 2020;7:4.

18. Health Commission of the Inner Mongolia Autonomous Region. Diagnosis and treatment of pneumonia caused by novel coronavirus by traditional Chinese medicine in the Inner Mongolia Autonomous region. 2020. Available at: http://wjw.wulanchabu.gov.cn/ information/wlcb_wjw52/msg22733261755.html.

19. Health Commission of Jiangsu Province. Traditional Chinese medicine differentiation and treatment protocol for novel coronavirus pneumonia in Jiangsu Province (Trial version 3). Jiangsu J Tradit Chin Med (Chin) 2020;52:1-2.

20. Military Medical Expert Group on the Front-line. Diagnosis and treatment protocol for COVID-19 from military medical team supporting Wuhan (Trial Version 2). Chin J Tuberc Respir Dis 2020;43:414-420.

21. Anhui Traditional Chinese Medicine Treatment Expert Team. Anhui Province expert consensus of new coronavirus pneumonia Chinese medicine treatment 2020. Available at: http://ahszyyxh.cn/news/ shownews.php?lang=cn\&id $=431$.

22. Sichuan Administration of Traditional Chinese Medicine. Suggested prescription of traditional Chinese medicine intervention for pneumonia caused by new coronavirus infection in Sichuan Province (1st Ed). 2020. Available at: http://www.sc.gov.cn/10462/1 2771/2020/1/25/0d37953ca2534d5382ba744e81725469.shtml.

23. Health Commission of Shandong province. Shandong Province new coronavirus pneumonia TCM diagnosis and treatment plan (2nd Ed). Available at: 2020. http://wsjkw.shandong.gov.cn/wzxxgk/ zfxxgk/gknr/tzwj/202003/t20200317_3315749.html.

24. Health Commission of Shanghai and Shanghai Administration of Traditional Chinese Medicine. Shanghai novel coronavirus pneumonia TCM diagnosis and treatment plan (Trial Second Edition). 2020. Available at: http://wsjkw.sh.gov.cn/ zyygz2/20200224/a1f1aab9745e4490867cb4aaf40eaado.html.

25. Health Commission of Hebei and Hebei Administration of TCM. TCM diagnosis and treatment plan of new coronavirus pneumonia in Hebei Province (Trial 2nd Ed). 2020. Available at: http://wsjkw. hebei.gov.cn/index.do?id=396244\&templet=content\&cid=202.

26. Mao J, Bi Y, Sun $\mathrm{H}$, et al. TCM diagnosis and treatment for novel coronavirus pneumonia of Tianjin Province. Tianjin J Tradit Chin Med (Chin) 2020 Available at: http://kns.cnki.net/kcms/ detail/12.1349.r.20200311.1245.002.html.

27. Health Commission of Liaoning Province. Treatment of pneumonia caused by novel coronavirus in Liaoning Province (Trial 2nd version). 2020. Available at: http://www.tieling.gov.cn/eportal/ui?pag eld $=195783 \&$ articleKey $=882985 \&$ columnld $=864756$.

28. Expert Group of Children's Pneumonia Collaborative Innovation Community of Chinese Medical Association, Expert group of Key Diseases in Pediatric Base of National Clinical Research Base of Traditional Chinese Medicine. Experts interpretation of traditional Chinese medicine treatment in diagnosis, treatment and prevention of corona virus disease 2019 in children: experts' consensus statement (Version 1). Chin Arch Tradit Chin Med (Chin) 2020. Available at: http://kns.cnki.net/kcms/detail/21.1546. R.20200302.1751.006.html.

29. Jiang $Y, L u X$, Jin R, et al. Expert consensus on the diagnosis of novel coronavirus infections in children (2nd Ed). Chin J Appl Clin Pediatr (Chin) 2020;35:143-150.

30. Hunan Administration of TCM. TCM diagnosis and treatment scheme of pneumonia caused by new coronavirus infection in Hunan Province (Trial Version 3). 2020. Available at: http://tcm. hunan.gov.cn/tcm/xxgk/tzgg/202002/t20200203_11168981.html.

31. Hu Z. Expert consensus on prevention and rehabilitation of integrated traditional Chinese and Western medicine during the outbreak of new coronavirus pneumonia in cancer patients in Hubei Province. Herald Med 2020. Available at: http://kns.cnki.net/kcms/ detail/42.1293.r.20200305.1155.004.html.

32. Li Y, Shi H. Expert consensus on diagnosis and treatment of novel coronavirus pneumonia in the elderly. J Chin Physic (Chin) 2020;22:161-165.

33. Pulmonary Disease Special Committee of Beijing Society of TCM. Expert guidance on the comprehensive intervention plan of TCM for the recovery of new coronavirus pneumonia (draft). Beijing $J$ Tradit Chin Med (Chin) 2020. Available at: http://kns.cnki.net/kcms/ detail/11.5635.r.20200221.1334.002.html.

34. Chinese Thoracic Society, Chinese Association of Chest Physicians. Guide for the prevention and treatment of coronavirus disease 2019 Chin J Tuberc Respir Dis (Chin) 2020. Available at: http://rs.yiigle. com/yufabiao/1187175.htm.

35. Health Commission of Taiyuan city. Guidelines for the prevention of pneumonia caused by novel coronavirus in Taiyuan City. 2020. Available at: http://wjw.taiyuan.gov.cn/doc/2020/02/01/954877.shtml

36. Health Commission of Henan Province. Novel coronavirus infection pneumonia prevention plan of Chinese Medicine in Henan Province. 2020. Available at: http://www.tcm.gov.cn/hydt/1700.htm.

37. Health Commission of Shandong province. Chinese Medicine Prevention Program for Pneumonia of Influenza and New Coronavirus Infections in Winter and Spring in Shandong Province in 2020. 2020. Available at: http://www.sdtcm.net/show-6-6531-1.html. 
38. Health Commission of Yunnan Province. TCM program for prevention and treatment of pneumonia infected by novel coronavirus in Yunnan Province (Trial 2nd Ed). 2020. Available at: http://www.pbh.yn.gov.cn/wjwWebsite/web/doc/ UU158140615448559052.

39. Health Commission of Xinjiang Uygur Autonomous Region. Prevention and treatment of pneumonia caused by novel coronavirus in Xinjiang Uygur Autonomous Region by TCM. 2020. Available at: http://www.xjbs.com.cn/zphd/2020-02/01/cms2223565article.shtml.

40. Administration of TCM of Tibet Autonomous Region. Tibetan medicine program for prevention and treatment of pneumonia caused by novel coronavirus infection in Tibet Autonomous Region (Trial 1st Ed). 2020. Available at: http://ex.chinadaily.com. cn/exchange/partners/82/rss/channel/cn/columns/j3u3t6/stories/ WS5e2f9dfca3107bb6b579bed2.html.

41. Health Commission of Qinghai Province. TCM prevention and treatment for pneumonia infected by novel coronavirus in Qinghai Province (Trial 2nd Ed). 2020. Available at: http://wsjkw.qinghai.gov. cn/zwgk/xxgkml/zcyy/2020/02/25/1582597802814.html.

42. Health Commission of Nanjing city. TCM prevention and treatment for pneumonia infected by novel coronavirus in Nanjing City (Trial Implementation). Jiangsu J Tradit Chin Med (Chin) 2020;52(4):9-10.

43. Guangdong Institute of Integrative Chinese and Western Medicine. Expert consensus on prevention and treatment of novel coronavirus pneumonia in guangdong with integrated traditional Chinese and Western medicine (trial version 1). World Chin Med (Chin) 2020. Available at: http://kns.cnki.net/kcms/detail/11.5529. r.20200311.1453.014.html.

44. Administration of TCM of Guizhou Province. Guizhou novel coronavirus pneumonia reference scheme for Chinese medicine prevention and control (2nd Edition). 2020. Available at: http://www. gz.xinhuanet.com/2020-02/19/c_1125595451.htm.

45. Health Commission of Hainan Province. Prevention and treatment of novel coronavirus infection in Hainan Province with TCM. 2020. Available at: http://www.hainan.gov.cn/hainan/5309/202002/fdea098 b24fa4499bb9c1b1a46ebafc1.shtml.

46. Ba Y, Li X, Min X, et al. Hubei Provincial Traditional Chinese Medical Hospital novel coronavirus pneumonia prevention and treatment plan of Chinese medicine (Third Edition). Hubei $\mathrm{J}$ Tradit Chin Med (Chin) 2020. Available at: http://kns.cnki.net/kcms/detail/42.1189. R.20200302.1503.003.html.

47. Chen G, Liu Y. TCM diagnosis and treatment plan and prevention plan of novel coronavirus pneumonia in Tongji Hospital Affiliated to Tongji Medical College of Huazhong University of science and technology. Herald Med 2020. Available at: http://kns.cnki.net/kcms/ detail/42.1293.r.20200211.1104.002.html.

48. The prevention and treatment of TCM of Hubei Hospital of TCM. The People's Government of Zaoyang; 2020. Available at: http:// www.zyzf.gov.cn/xwzx/gjj/202001/t20200125_2017327.shtml

49. Professional Commission of geriatric medicine of Hubei society of TCM. Expert consensus on prevention and treatment of novel coronavirus pneumonia in the elderly (First Edition). Hubei J Tradit Chin Med (Chin). 2020. Available at: http://kns.cnki.net/kcms/ detail/42.1189.r.20200228.1123.002.html.

50. Health Commission of Heilongjiang Province. Prevention and treatment of pneumonia caused by novel coronavirus in Heilongjiang Province (2nd Ed). 2020. Available at: https://m.dbw.cn/heilongjiang/ system/2020/02/02/058330150.shtml.

51. Jiangxi TCM Treatment Expert. TCM prevention and treatment program of new coronavirus pneumonia in Jiangxi Province. 2020. Available at: http://www.jxhfpc.gov.cn/doc/2020/01/25/137657.shtml.

52. Administration of TCM of Ningxia Hui Autonomous Region. Prevention and treatment of pneumonia caused by new coronavirus infection in Ningxia Hui Autonomous Region (Trial). 2020. Available at: http://wsjkw.nx.gov.cn/info/1040/13360.htm.

53. Tianjin TCM Expert Group. Prevention and treatment program of TCM for novel coronavirus pneumonia in Tianjin (Trial Third Edition). 2020. Available at: http://www.tjdl.gov.cn/zwgk/ system/2020/01/27/004044626.shtml.

54. Beijing Administration of Traditional Chinese Medicine. Traditional
Chinese medicine prevention and treatment program for novel coronavirus pneumonia in Beijing (Trial Version 4). Beijing J Tradit Chin Med (Chin) 2020. Available at: http://kns.cnki.net/kcms/ detail/11.5635.R.20200316.1713.004.html.

55. Health Commission of Gansu Province. Prevention and treatment of pneumonia caused by new coronavirus infection in Gansu Province (Trial 2nd Ed). 2020. Available at: http://wsjk.gansu.gov. $\mathrm{cn} /$ single/10991/83576.html.

56. Zou B, Li M, Fan T, et al. Summary of experience in the treatment of severe COVID-19 with TCM and suggestions on treatment plan. J Tradit Chin Med (Chin) 2020. Available at: http://kns.cnki.net/kcms/ detail/11.2166.r.20200402.1149.002.html.

57. Health Commission of Shaanxi province. TCM Treatment for pneumonia infected by novel coronavirus in Shaanxi Province (Trial 2nd Edition). 2020. Available at: http://sxwjw.shaanxi.gov.cn/ art/2020/2/2/art_10_67602.html.

58. Lin L, Chen $\mathrm{Y}$, Tan $\mathrm{Y}$, et al. TCM treatment plan for novel coronavirus pneumonia in Guangdong Province (Trial version 2). J Tradit Chin Med (Chin) 2020. Available at: http://kns.cnki.net/kcms/ detail/11.2166.R.20200302.0930.004.html.

59. Health Commission of Guangxi Zhuang Autonomous Region. Treatment of novel coronavirus pneumonia in the Guangxi Zhuang Autonomous Region with Chinese medicine (Trial). 2020. Available at: http://www.gxyz120.com/show.php?contentid=2410.

60. Health Commission of Jilin Province. Treatment of novel coronavirus pneumonia in Jilin province by TCM (First Edition). 2020. Available at: http://www.jl.gov.cn/szfzt/jlzxd/tzgg/202001/t20200126_6654831.html.

61. Xue B. How does Qingfei Paidu Decoction work quickly and effectively 2020 Available from: http://www.satcm.gov.cn/xinxifabu/ meitibaodao/2020-03-01/13529.html.

62. Yu P, Li Y, Wan S, et al. Clinical observation of therapeutic effect of Lianhua Qingwen Granule combined with abidor on mild coronavirus pneumonia. Chin Pharm J (Chin) 2020. Available at: http://kns.cnki. $\mathrm{net} / \mathrm{kcms} /$ detail/11.2162.R.20200422.1429.002.html.

63. Lv R, Wang W, li X. Clinical observation on 63 suspected cases of coronavirus pneumonia treated by Lianhua Qingwen Granule Combined with Western medicine conventional therapy. J Tradit Chin Med (Chin) 2020;61:655-659.

64. Cheng D, Wang W, Li Y, et al. Efficacy analysis of 51 COVID-19 patients treated with Chinese medicine Lianhua Qingwen: a multicenter retrospective study. Tianjin J Tradit Chin Med (Chin) 2020:1-6.

65. Liu M, Gao Y, Yuan Y, et al. Efficacy and safety of integrated Traditional Chinese and Western medicine for corona virus disease 2019 (COVID-19): a systematic review and meta-analysis. Pharmacol Res 2020:104896.

66. Wang R, Yang S, Xie C, et al. Clinical observation of Qingfeipaidu Decoction in the treatment of COVID-19. Pharm Clin Chin Mater Med (Chin) 2020;36:13-18.

67. Luo E, Zhang D, Luo $\mathrm{H}$, et al. Treatment efficacy analysis of traditional Chinese medicine for novel coronavirus pneumonia (COVID-19): an empirical study from Wuhan, Hubei Province, China. Chin Med 2020;15:34.

68. Ye Y. The G-CHAMPS Collaborative Group. Guideline-based Chinese herbal medicine treatment plus standard care for severe coronavirus disease 2019 (G-CHAMPS): evidence from China. Front Med 2020;7:256.

69. Li Y, Liu X, Guo L, et al. CHM for treating novel coronavirus (COVID-19) pneumonia: protocol for a systematic review and metaanalysis. System Rev 2020;9:75.

70. Wu D, Jiang W, Yu L, et al. Quality assessment of clinical practice guidelines for infectious diseases in China. J Evid Based Med (Chin) 2018;11:95-100.

71. Ang L, Lee HW, Choi JY, et al. Herbal medicine and pattern identification for treating COVID-19: a rapid review of guidelines. Integr Med Res 2020;9:100407.

72. Ang L, Lee HW, Kim A, et al. Herbal medicine for treatment of children diagnosed with COVID-19: a review of guidelines. Complement Ther Clin Pract 2020;39:101174.

(Accepted August 24, 2020) Edited by YUAN Lin 